\title{
NANOGrav Hints to Primordial Black Holes as Dark Matter
}

\author{
V. De Luca, ${ }^{1, *}$ G. Franciolini, ${ }^{1, \dagger}$ and A. Riotto ${ }^{1,2, \ddagger}$ \\ ${ }^{1}$ Département de Physique Théorique and Centre for Astroparticle Physics (CAP), \\ Université de Genève, 24 quai E. Ansermet, CH-1211 Geneva, Switzerland \\ ${ }^{2}$ INFN, Sezione di Roma, Piazzale Aldo Moro 2, 00185, Roma, Italy
}

(Dated: Wednesday $25^{\text {th }}$ November, 2020)

\begin{abstract}
The NANOGrav Collaboration has recently published a strong evidence for a stochastic commonspectrum process that may be interpreted as a stochastic gravitational wave background. We show that such a signal can be explained by second-order gravitational waves produced during the formation of primordial black holes from the collapse of sizeable scalar perturbations generated during inflation. This possibility has two predictions: i) the primordial black holes may comprise the totality of the dark matter with the dominant contribution to their mass function falling in the range $\left(10^{-15} \div 10^{-11}\right) M_{\odot}$ and $i$ i) the gravitational wave stochastic background will be seen as well by the LISA experiment.
\end{abstract}

Introduction. The NANOGrav Collaboration has recently published an analysis of 12.5 yrs of pulsar timing data [1] reporting a strong evidence for a stochastic common-spectrum process. The latter may be compatible with a Gravitational Wave (GW) signal with strain amplitude $\sim 10^{-15}$ at a frequency $f \sim 3 \cdot 10^{-8} \mathrm{~Hz}$ with an almost flat $\mathrm{GW}$ spectrum, $\Omega_{\mathrm{GW}}(f) \sim f^{(-1.5 \div 0.5)}$ at $1 \sigma$ level. In particular, their analysis shows the presence of a stochastic process across fourty-five pulsars which can be interpreted in terms of a common-spectrum process strongly preferred against independent red-noise signals. Despite being in partial contrast with some other bounds on the stochastic background of GWs, the NANOGrav Collaboration stresses that the detected signal is due to an improved treatment of the intrinsic pulsar red noise. On the other side, it is important to stress that the NANOGrav Collaboration does not claim a detection of GWs since the signal does not possess quadrupole correlations.

The goal of this paper is to show that the NANOGrav signal, if interpreted as a GW background, can be naturally explained by a flat spectrum of GWs inevitably generated at second-order in perturbation theory during the formation of Primordial Black Holes (PBHs) in the case in which the latter form from the collapse of large curvature perturbation generated during inflation upon horizon re-entry (see also Refs. [2, 3] for reviews on PBHs physics and Ref. [4] for a review on the constraints on their abundance).

Two nice by-products of this explanation are that i) the dominant contribution to the PBH mass function falls in the range $\left(10^{-15} \div 10^{-11}\right) M_{\odot}$ where the $\mathrm{PBHs}$ can comprise the totality of the dark matter in the universe; ii) the GW stochastic spectrum propagates to frequencies testable by future experiments, such as LISA [5].

The PBH abundance. The most common formation scenario for PBHs is through an enhancement of the power spectrum of the comoving curvature perturbation $\zeta$ during inflation, at scales much smaller than those probed by CMB observations [6-8]. During the radiationdominated phase, an overdense region collapses to form a $\mathrm{PBH}$ at horizon re-entry if the volume-averaged density contrast is larger than a critical value $\delta_{c}$, which has been found with dedicated relativistic numerical simulations in Refs. [9-11].

We define the comoving curvature perturbation power spectrum as

$$
\left\langle\zeta\left(\vec{k}_{1}\right) \zeta\left(\vec{k}_{2}\right)\right\rangle^{\prime}=\frac{2 \pi^{2}}{k_{1}^{3}} \mathcal{P}_{\zeta}\left(k_{1}\right)
$$

where we have adopted the standard prime notation indicating that we do not explicitly write down the $(2 \pi)^{3}$ times the Dirac delta of momentum conservation. In comoving slices, the overdensity is usually expressed in terms of the curvature perturbation through the nonlinear relation [11]

$$
\delta(\vec{x})=-\frac{8}{9 a^{2} H^{2}} e^{-5 \zeta(\vec{x}) / 2} \nabla^{2} e^{\zeta(\vec{x}) / 2},
$$

where $a$ is the scale factor and $H=\dot{a} / a$ the Hubble rate.

Assuming Gaussian curvature perturbations, one can estimate the mass fraction of the Universe that collapses to form PBHs at formation by computing the probability $P(\delta)$ that the overdensity is larger than the critical threshold following the Press-Schechter formalism as [2]

$$
\beta\left(M_{\mathrm{PBH}}\right)=\int_{\delta_{c}}^{\infty} \mathrm{d} \delta \frac{M_{\mathrm{PBH}}}{M_{H}} P(\delta),
$$

where one has to keep into account the scaling law relating the PBH mass to the horizon mass $M_{H}$ for overdensities close to the critical threshold for collapse as [12-14]

$$
M_{\mathrm{PBH}}=\kappa M_{H}\left(\delta-\delta_{c}\right)^{\gamma_{c}}
$$

in terms of the constants $\kappa=3.3$ and $\gamma_{c}=0.36$ in a radiation-dominated universe [15-20]. The horizon mass $M_{H}$ is related to the characteristic comoving frequency of the perturbation as

$$
M_{H} \simeq 33\left(\frac{10^{-9} \mathrm{~Hz}}{f}\right)^{2} M_{\odot}
$$


The variance of the density field $\delta(\vec{x})$ is given by

$$
\sigma_{\delta}^{2}=\int_{0}^{\infty} \mathrm{d} \ln k T^{2}\left(k, R_{H}\right) W^{2}\left(k, R_{H}\right) \mathcal{P}_{\delta}(k)
$$

in terms of the density power spectrum $\mathcal{P}_{\delta}(k)$. A real space top-hat window function $W\left(k, R_{H}\right)$ is introduced to smooth out the density contrast on the comoving horizon length $R_{H} \sim 1 / a H$, given by ${ }^{1}$

$$
W\left(k, R_{H}\right)=3 \frac{\sin \left(k R_{H}\right)-\left(k R_{H}\right) \cos \left(k R_{H}\right)}{\left(k R_{H}\right)^{3}},
$$

and the transfer function during radiation domination with constant degrees of freedom is provided by

$$
T\left(k, R_{H}\right)=3 \frac{\sin \left(k R_{H} / \sqrt{3}\right)-\left(k R_{H} / \sqrt{3}\right) \cos \left(k R_{H} / \sqrt{3}\right)}{\left(k R_{H} / \sqrt{3}\right)^{3}} .
$$

Notice that, in our results, we have accounted for the ineludible non-Gaussianity inherited by the non-linear relation between the curvature perturbation and the density contrast as shown in Eq. (2), see Refs. [22, 23].

To assess if PBHs may or not represent the dark matter in the universe, one usually introduces the $\mathrm{PBH}$ mass function mass $f_{\mathrm{PBH}}\left(M_{\mathrm{PBH}}\right)$ as the fraction of $\mathrm{PBHs}$ with $M_{\mathrm{PBH}}[2]$

$$
f_{\mathrm{PBH}}\left(M_{\mathrm{PBH}}\right)=\frac{1}{\Omega_{\mathrm{DM}}} \frac{\mathrm{d} \Omega_{\mathrm{PBH}}}{\mathrm{d} \ln M_{\mathrm{PBH}}},
$$

such that the total fraction of dark matter in the form of $\mathrm{PBHs}$ is given by

$$
f_{\mathrm{PBH}}=\int f_{\mathrm{PBH}}\left(M_{\mathrm{PBH}}\right) \mathrm{d} \ln M_{\mathrm{PBH}} .
$$

After matter-radiation equality, it can be expressed in terms of the mass fraction $\beta$ as (see for example [24])

$$
f_{\mathrm{PBH}}\left(M_{\mathrm{PBH}}\right)=\frac{1}{\Omega_{\mathrm{DM}}}\left(\frac{M_{\mathrm{eq}}}{M_{\mathrm{PBH}}}\right)^{1 / 2} \beta\left(M_{\mathrm{PBH}}\right),
$$

where the overall factor, dependent on the horizon mass at matter-radiation equality $M_{\text {eq }}=2.8 \cdot 10^{17} M_{\odot}$, accounts for the energy density evolution during the remaining radiation-dominated phase after formation of $\mathrm{PBHs}$ of $\operatorname{mass} M_{\mathrm{PBH}}$.

In the following sections, we will show that a signal as the one observed by NANOGrav naturally arises from a class of models with a broad and flat power spectrum of the curvature perturbation of the form [25]

$$
\mathcal{P}_{\zeta}(k) \approx A_{\zeta} \Theta\left(k_{s}-k\right) \Theta\left(k-k_{l}\right), \quad k_{s} \gg k_{l}
$$

\footnotetext{
1 As pointed out in Ref. [21], the uncertainty in the choice of the window function is reduced if the same smoothing is adopted in the calculation of the threshold. For such a reason we have chosen $\delta_{c}=0.51$, see Tab. I in Ref. [21].
}

where $\Theta$ is the Heaviside step function and $A_{\zeta}$ is the amplitude of the power spectrum. This shape may be generated naturally for modes which exit the Hubble radius during a non-attractor phase, obtained through an ultra slow-roll regime of the inflaton potential, as a result of a duality transformation which maps the non-attractor phase into a slow-roll phase [26-29].

The power spectrum of GWs. To investigate the GW signal, we first define the linearized line element in tightly-coupled radiation domination as

$\mathrm{d} s^{2}=a^{2}\left\{-(1+2 \Psi) \mathrm{d} \eta^{2}+\left[(1-2 \Psi) \delta_{i j}+\frac{h_{i j}}{2}\right] \mathrm{d} x^{i} \mathrm{~d} x^{j}\right\}$,

in terms of the Newtonian-gauge scalar metric perturbation $\Psi$ and the transverse-traceless tensor metric perturbation $h_{i j}$. Focusing on the signal sourced at secondorder by linear scalar perturbations [30-47], one can expand the Einstein's equations and determine the equation of motion for the GWs as

$$
h_{i j}^{\prime \prime}+2 \mathcal{H} h_{i j}^{\prime}-\nabla^{2} h_{i j}=-4 \mathcal{T}_{i j}^{\ell m} \mathcal{S}_{\ell m}
$$

where' is the derivative with respect to the conformal time $\eta$ and $\mathcal{T}_{i j}{ }^{\ell m}$ projects the source term $\mathcal{S}_{\ell m}$ into its transverse and traceless part. In the radiation phase the source is given by [30]

$$
\mathcal{S}_{i j}=2 \partial_{i} \partial_{j}\left(\Psi^{2}\right)-2 \partial_{i} \Psi \partial_{j} \Psi-\partial_{i}\left(\frac{\Psi^{\prime}}{\mathcal{H}}+\Psi\right) \partial_{j}\left(\frac{\Psi^{\prime}}{\mathcal{H}}+\Psi\right),
$$

while the projector in Fourier space using the chiral basis is

$$
\widetilde{\mathcal{T}}_{i j}^{\ell m}(\vec{k})=e_{i j}^{\mathrm{L}}(\vec{k}) \otimes e^{\mathrm{L} \ell m}(\vec{k})+e_{i j}^{\mathrm{R}}(\vec{k}) \otimes e^{\mathrm{R} \ell m}(\vec{k}),
$$

where $e_{i j}^{\mathrm{L}, \mathrm{R}}$ are the polarisation tensors. In Eq. (15) the scalar perturbation $\Psi(\eta, \vec{k})$ can be expressed in terms of the comoving curvature perturbation as [48]

$$
\Psi(\eta, \vec{k}) \equiv \frac{2}{3} T(k \eta) \zeta(\vec{k}) .
$$

The current abundance of GWs is found to be [49]

$$
\begin{aligned}
\frac{\Omega_{\mathrm{GW}}(f)}{\Omega_{r, 0}} & =\frac{c_{g}}{972} \iint_{\mathcal{S}} \mathrm{d} x \mathrm{~d} y \frac{x^{2}}{y^{2}}\left[1-\frac{\left(1+x^{2}-y^{2}\right)^{2}}{4 x^{2}}\right]^{2} \\
& \times \mathcal{P}_{\zeta}(k x) \mathcal{P}_{\zeta}(k y) \mathcal{I}^{2}(x, y),
\end{aligned}
$$

where the integration region $\mathcal{S}$ extends to $x>0$ and to $|1-x| \leq y \leq 1+x$, and $k=2 \pi f$. The parameter $c_{g}$ defined as

$$
c_{g} \equiv \frac{g_{*}\left(M_{H}\right)}{g_{*}^{0}}\left(\frac{g_{* S}^{0}}{g_{* S}\left(M_{H}\right)}\right)^{4 / 3}
$$

accounts for the change of the effective degrees of freedom of the thermal radiation $g_{*}$ and $g_{* S}$ (where the superscript ${ }^{0}$ indicates the values today) during the evolution 

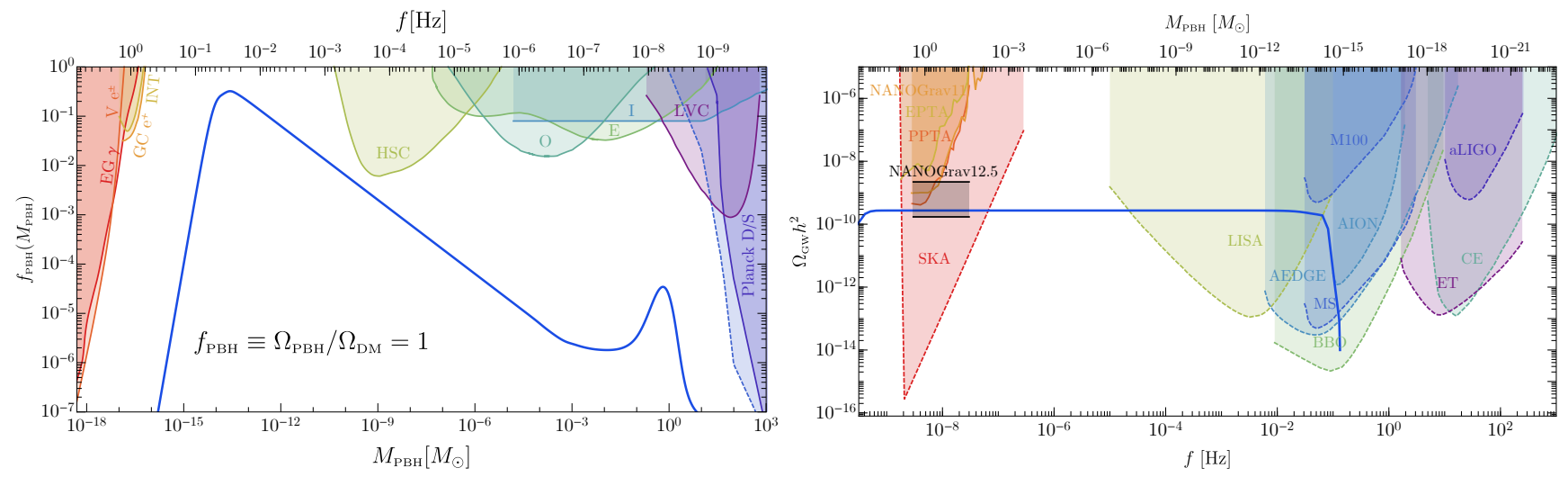

FIG. 1. Left: Mass function resulting from a flat power spectrum such that it peaks at $\simeq 10^{-14} M_{\odot}$, with $A_{\zeta} \simeq 5.8 \cdot 10^{-3}$ and $k_{s}=10^{9} k_{l} \simeq 1.6 \mathrm{~Hz}$, and PBHs comprise the totality of DM, i.e. $f_{\mathrm{PBH}}=1$. In the tail of the population, around $M_{\odot}$, one can notice the bump in the PBH production due to the decrease of the threshold by QCD epoch equation of state $[24,51]$. Shown are the most stringent constraints in the mass range of phenomenological interest coming from the Hawking evaporation producing extra-galactic gamma-ray (EG $\gamma$ ) [52], $e^{ \pm}$observations by Voyager 1 ( $\mathrm{V} e^{ \pm}$) [53], positron annihilations in the Galactic Center (GC $e^{+}$) [54] and gamma-ray observations by INTEGRAL (INT) [55] (for other constraints in this mass range see also [56-61]), microlensing searches by Subaru HSC [62, 63], MACHO/EROS [64, 65], Ogle [66] and Icarus [67], and those coming from CMB distortions by spherical or disk accretion (Planck S and Planck D, respectively) [68, 69]. LVC stands for the constraint coming from LIGO/Virgo Collaboration measurements [70-72]. We neglect the role of accretion which has been shown to affect constraints on masses larger than $\mathcal{O}(10) M_{\odot}$ [73, 74]. See Ref. [4] for a comprehensive review on constraints on the PBH abundance. Notice that there are no stringent constraints in the PBH mass range of interest [75, 76]. Right: The abundance of GWs according to our scenario. In black the $95 \%$ C.I. from the NANOGrav 12.5 yrs experiment is shown. For more details about the projected sensitivities see the main text.

(assuming Standard Model physics), and it is of order $c_{g}=0.4$ for modes related to the formation of asteroidmass PBHs. Also, $\Omega_{r, 0}$ stands for the current radiation density if the neutrinos were massless, $\mathcal{I}^{2} \equiv \mathcal{I}_{c}^{2}+\mathcal{I}_{s}^{2}$ and

$$
\begin{aligned}
& \mathcal{I}_{c}(x, y)=4 \int_{0}^{\infty} \mathrm{d} \tau \tau(-\sin \tau)[2 T(x \tau) T(y \tau) \\
& \left.+\left(T(x \tau)+x \tau T^{\prime}(x \tau)\right)\left(T(y \tau)+y \tau T^{\prime}(y \tau)\right)\right],
\end{aligned}
$$

$\mathcal{I}_{s}(x, y)$ being the same function, but with $(-\sin \tau)$ replaced by $(\cos \tau)$, see Refs. [49, 50].

Results. We have collected our results in Fig. 1. In the left panel, we have plotted the mass function corresponding to the primordial curvature perturbation given in Eq. (12). As described in Ref. [25], the peak of the mass function for a broad flat spectrum (12) corresponds to the mass inside the horizon when the shortest scale $\sim 1 / k_{s}$ re-enters the horizon. At smaller masses, the mass function goes as $M_{\mathrm{PBH}}^{3.8}$ due to the dinamics of the critical collapse, while at larger masses falls down as $\sim M_{\mathrm{PBH}}^{-3 / 2}$ and has a sub-dominant peak around $\sim M_{\odot}$ due to the change of equation of state during the QCD phase transition [24,51]. Given the absence of constraints in the mass range of support of the $\mathrm{PBH}$ mass function ( the femtolensing bounds have been shown to be inconsistent once the extended nature of the source as well as wave optics effects are properly taken into account $[75,76])$, the integral of the latter can be chosen in such a way that the PBHs contribute to the totality of the dark matter, that is

$$
f_{\mathrm{PBH}}=\int f_{\mathrm{PBH}}\left(M_{\mathrm{PBH}}\right) \mathrm{d} \ln M_{\mathrm{PBH}}=1 .
$$

As a consequence the first prediction of our scenario is that the signal seen by NANOGrav, if interpreted as a stochastic background of GWs produced as second-order within the $\mathrm{PBH}$ model, is in agreement with the possibility that all the dark matter is in the form of extremely light $\mathrm{PBH}$.

On the right panel of Fig. 1, we show the corresponding spectrum of the second-order GW abundance as a function of the frequency which falls within the $95 \%$ C.I. from the NANOGrav 12.5 yrs observation. Shown are the constraints coming from experiment EPTA [77], PPTA [78], NANOGrav 11 yrs [79, 80] and future sensitivity curves for planned experiments like SKA [81], LISA [5] (power-law integrated sensitivity curve expected to fall in between the designs named $\mathrm{C} 1$ and $\mathrm{C} 2$ in Ref. [82]), DECIGO/BBO [83], CE [84], Einstein Telescope [85, 86], Advanced Ligo + Virgo collaboration [87], Magis-space and Magis-100 [88], AEDGE [89] and AION [90]. Notice that a portion of the $95 \%$ C.I. of NANOGrav $12.5 \mathrm{yrs}$ is in tension with NANOGrav 11 yrs and PPTA. However, according to the NANOGrav Collaboration [1] the improved priors for the intrinsic pulsar red noise used in the novel analysis relaxes the NANOGrav 11 yrs bound. Nevertheless, the predicted signal within our scenario falls below all bounds. The GW abundance spectrum propagates flat entering the LISA detectable region and de- 
cays rapidly at the frequency corresponding to the shortest scale $1 / k_{s}$. The second prediction of our scenario is therefore that the second-order GWs seen by NANOGrav should also be detected by the forthcoming experiment LISA, and eventually MS and BBO as well. Notice also that the present scenario is consistent with the candidate event found by the HSC collaboration and discussed in Ref. [91].

Both predictions of the scenario described in this paper depend only on the choice of the shortest scale $1 / k_{s}$ and the requirement of the $\mathrm{PBH}$ abundance being equal to the dark matter one.

Conclusions. The discovery of a primordial stochastic background of GWs would be another fundamental pillar in GW astronomy. In this Letter, we have shown that the recently published stochastic common-spectrum process by the NANOGrav Collaboration, if interpreted as an indication of a GW background, can be naturally linked to the physics of PBHs. Indeed, the formation of $\mathrm{PBH}$ in the early universe due to the collapse of sizeable overdensities generated during inflation is inevitably accompanied by the generation of GWs. Interestingly enough, the NANOGrav observation is consistent with a mass range of $\mathrm{PBHs}$ such that the latter can comprise the totality of the dark matter. Furthermore, the GW signal is characterized by a flat spectrum which will make it visibile even at much larger frequencies, most notably by the forthcoming experiment LISA.

Note Added. When completing this work, other interpretations of the NANOGrav signal have appeared other than through super-massive BH coalescences [92]. Refs. $[93,94]$ point out the possible generation of GWs through cosmic strings. Ref. [95] discusses as well the possibility of explaining the NANOGrav signal with second-order GWs related to the $\mathrm{PBH}$ scenario. They identify a mass range of PBHs providing the seeds of supermassive BHs which cannot be the dark matter. Contrarily to that work, in our scenario the PBH masses are much smaller and PBHs can comprise the totality of the dark matter. The possible confirmation of the scenario proposed in this work by future HSC observations has been forcasted in Ref. [96].

Acknowledgments. We thank Ranjan Laha, Marek Lewicki and Julian Muñoz for discussions. V.DL., G.F. and A.R. are supported by the Swiss National Science Foundation (SNSF), project The Non-Gaussian Universe and Cosmological Symmetries, project number: 200020178787 .

\footnotetext{
*Valerio.DeLuca@unige.ch

† Gabriele.Franciolini@unige.ch

‡ Antonio.Riotto@unige.ch
}

[1] Z. Arzoumanian et al. [NANOGrav], [astro-ph.HE/2009.04496].

[2] M. Sasaki, T. Suyama, T. Tanaka and S. Yokoyama, Class. Quant. Grav. 35, no. 6, 063001 (2018) [astro-ph.C0/1801.05235].

[3] A. M. Green and B. J. Kavanagh, [astro-ph.CO/2007.10722].

[4] B. Carr, K. Kohri, Y. Sendouda and J. Yokoyama, [astro-ph.Co/2002.12778].

[5] H. Audley et al., [astro-ph. IM/1702.00786].

[6] P. Ivanov, P. Naselsky and I. Novikov, Phys. Rev. D 50, 7173 (1994).

[7] J. García-Bellido, A.D. Linde and D. Wands, Phys. Rev. D 54 (1996) 6040 [astro-ph/9605094].

[8] P. Ivanov, Phys. Rev. D 57, $7145 \quad$ (1998) [astro-ph/9708224].

[9] I. Musco, Phys. Rev. D 100 (2019) no.12, 123524 [gr-qc/1809.02127].

[10] C. Germani and I. Musco, Phys. Rev. Lett. 122 (2019) no.14, 141302 [astro-ph.CO/1805.04087].

[11] T. Harada, C. M. Yoo, T. Nakama and Y. Koga, Phys. Rev. D 91 (2015) no.8, 084057 [gr-qc/1503.03934].

[12] M. W. Choptuik, Phys. Rev. Lett. 70 (1993), 9-12

[13] C. R. Evans and J. S. Coleman, Phys. Rev. Lett. 72 (1994), 1782-1785 [gr-qc/9402041].

[14] J. C. Niemeyer and K. Jedamzik, Phys. Rev. Lett. 80 (1998), 5481-5484 [astro-ph/9709072].

[15] T. Koike, T. Hara and S. Adachi, Phys. Rev. Lett. 74, 5170 (1995) [gr-qc/9503007].

[16] I. Musco, J. C. Miller and L. Rezzolla, Class. Quant. Grav. 22, 1405 (2005) [gr-qc/0412063].

[17] I. Musco, J. C. Miller and A. G. Polnarev, Class. Quant. Grav. 26, 235001 (2009) [gr-qc/0811.1452].

[18] I. Musco and J. C. Miller, Class. Quant. Grav. 30, 145009 (2013) [gr-qc/1201.2379].

[19] A. Kalaja, N. Bellomo, N. Bartolo, D. Bertacca, S. Matarrese, I. Musco, A. Raccanelli and L. Verde, JCAP 10 (2019), 031 [astro-ph. C0/1908.03596].

[20] A. Escrivà, Phys. Dark Univ. 27 (2020), 100466 [gr-qc/1907.13065].

[21] S. Young, Int. J. Mod. Phys. D 29 (2019) no.02, 2030002 [astro-ph.CO/1905.01230].

[22] V. De Luca, G. Franciolini, A. Kehagias, M. Peloso, A. Riotto and C. Ünal, JCAP 07 (2019), 048 [astro-ph.C0/1904.00970].

[23] S. Young, I. Musco and C. T. Byrnes, JCAP 11 (2019), 012 [astro-ph.C0/1904.00984].

[24] C. T. Byrnes, M. Hindmarsh, S. Young and M. R. S. Hawkins, JCAP 08 (2018), 041 [astro-ph.C0/1801.06138].

[25] V. De Luca, G. Franciolini and A. Riotto, Phys. Lett. B 807 (2020), 135550 [astro-ph.Co/2001.04371].

[26] D. Wands, Phys. Rev. D 60 (1999), 023507 [gr-qc/9809062].

[27] S. M. Leach and A. R. Liddle, Phys. Rev. D 63 (2001), 043508 [astro-ph/0010082].

[28] S. M. Leach, M. Sasaki, D. Wands and A. R. Liddle, Phys. Rev. D 64 (2001), 023512 [astro-ph/0101406].

[29] M. Biagetti, G. Franciolini, A. Kehagias and A. Riotto, JCAP 07 (2018), 032 [astro-ph.CO/1804.07124].

[30] V. Acquaviva, N. Bartolo, S. Matarrese and A. Riotto, Nucl. Phys. B 667 (2003) 119 [astro-ph/0209156].

[31] S. Mollerach, D. Harari and S. Matarrese, Phys. Rev. D 69 (2004) 063002 [astro-ph/0310711]. 
[32] K. N. Ananda, C. Clarkson and D. Wands, Phys. Rev. D 75 (2007) 123518 [gr-qc/0612013].

[33] D. Baumann, P. J. Steinhardt, K. Takahashi and K. Ichiki, Phys. Rev. D 76 (2007) 084019 [hep-th/0703290].

[34] R. Saito and J. Yokoyama, Prog. Theor. Phys. 123, 867 (2010) Erratum: [Prog. Theor. Phys. 126, 351 (2011)] [astro-ph.c0/0912.5317].

[35] J. Garcia-Bellido, M. Peloso and C. Unal, JCAP 12 (2016), 031 [astro-ph.C0/1610.03763].

[36] R. g. Cai, S. Pi and M. Sasaki, Phys. Rev. Lett. 122 (2019) no.20, 201101 [astro-ph.co/1810.11000].

[37] N. Bartolo, V. De Luca, G. Franciolini, M. Peloso, D. Racco and A. Riotto, Phys. Rev. D 99 (2019) no.10, 103521 [astro-ph.CO/1810.12224].

[38] N. Bartolo, V. De Luca, G. Franciolini, A. Lewis, M. Peloso and A. Riotto, Phys. Rev. Lett. 122 (2019) no.21, 211301 [astro-ph.C0/1810.12218].

[39] C. Unal, Phys. Rev. D 99 (2019) no.4, 041301 [astro-ph.C0/1811.09151].

[40] S. Wang, T. Terada and K. Kohri, Phys. Rev. D 99 (2019) no.10, 103531 [astro-ph.CO/1903.05924].

[41] R. G. Cai, S. Pi, S. J. Wang and X. Y. Yang, JCAP 10 (2019), 059 [astro-ph.C0/1907.06372].

[42] N. Bartolo, D. Bertacca, V. De Luca, G. Franciolini, S. Matarrese, M. Peloso, A. Ricciardone, A. Riotto and G. Tasinato, JCAP 02 (2020), 028 [astro-ph.C0/1909.12619].

[43] V. De Luca, G. Franciolini, A. Kehagias and A. Riotto, JCAP 03 (2020), 014 [gr-qc/1911.09689].

[44] K. Inomata and T. Terada, Phys. Rev. D 101 (2020) no.2, 023523 [gr-qc/1912.00785].

[45] C. Yuan, Z. C. Chen and Q. G. Huang, Phys. Rev. D 101 (2020) no.6, 063018 [astro-ph.Co/1912.00885].

[46] S. Pi and M. Sasaki, [gr-qc/2005.12306].

[47] C. Yuan and Q. G. Huang, [astro-ph.Co/2007.10686].

[48] D.H. Lyth and A. Riotto, Phys. Rept. 314 (1999) 1 [hep-ph/9807278].

[49] J. R. Espinosa, D. Racco and A. Riotto, JCAP 1809, no. 09, 012 (2018) [hep-ph/1804.07732].

[50] K. Kohri and T. Terada, Phys. Rev. D 97 (2018) no.12, 123532 [gr-qc/1804.08577].

[51] K. Jedamzik, Phys. Rev. D 55 (1997), 5871-5875 [astro-ph/9605152].

[52] A. Arbey, J. Auffinger and J. Silk, Phys. Rev. D 101 (2020) no.2, 023010 [astro-ph.Co/1906.04750].

[53] M. Boudaud and M. Cirelli, Phys. Rev. Lett. 122 (2019) no.4, 041104 [astro-ph.HE/1807.03075].

[54] W. DeRocco and P. W. Graham, Phys. Rev. Lett. 123 (2019) no.25, 251102 [astro-ph.C0/1906.07740].

[55] R. Laha, J. B. Muñoz and T. R. Slatyer, Phys. Rev. D 101 (2020) no.12, 123514 [astro-ph.C0/2004.00627].

[56] B. J. Carr, K. Kohri, Y. Sendouda and J. Yokoyama, Phys. Rev. D 81 (2010), 104019 [astro-ph.C0/0912.5297].

[57] G. Ballesteros, J. Coronado-Blázquez and D. Gaggero, Phys. Lett. B $\mathbf{8 0 8}$ (2020), 135624 [astro-ph.C0/1906.10113].

[58] R. Laha, Phys. Rev. Lett. 123 (2019) no.25, 251101 [astro-ph.HE/1906.09994].

[59] H. Poulter, Y. Ali-Haïmoud, J. Hamann, M. White and A. G. Williams, [astro-ph.C0/1907.06485].

[60] B. Dasgupta, R. Laha and A. Ray, Phys. Rev. Lett. 125 (2020) no.10, 101101 [hep-ph/1912.01014].
[61] R. Laha, P. Lu and V. Takhistov, [astro-ph.C0/2009.11837].

[62] H. Niikura, M. Takada, N. Yasuda, R. H. Lupton, T. Sumi, S. More, T. Kurita, S. Sugiyama, A. More, M. Oguri and M. Chiba, Nature Astron. 3 (2019) no.6, 524-534 [astro-ph.C0/1701.02151].

[63] N. Smyth, S. Profumo, S. English, T. Jeltema, K. McKinnon and P. Guhathakurta, Phys. Rev. D 101 (2020) no.6, 063005 [astro-ph.Co/1910.01285].

[64] C. Alcock et al. [MACHO], Astrophys. J. Suppl. 136 (2001), 439-462 [astro-ph/0003392].

[65] R. A. Allsman et al. [Macho], Astrophys. J. Lett. 550 (2001), L169 [astro-ph/0011506]

[66] H. Niikura, M. Takada, S. Yokoyama, T. Sumi and S. Masaki, Phys. Rev. D 99 (2019) no.8, 083503 [astro-ph.CO/1901.07120].

[67] M. Oguri, J. M. Diego, N. Kaiser, P. L. Kelly and T. Broadhurst, Phys. Rev. D 97 (2018) no.2, 023518 [astro-ph.Co/1710.00148].

[68] Y. Ali-Haïmoud and M. Kamionkowski, Phys. Rev. D 95 (2017) no.4, 043534 [astro-ph.C0/1612.05644].

[69] P. D. Serpico, V. Poulin, D. Inman and K. Kohri, Phys. Rev. Res. 2 (2020) no.2, 023204 [astro-ph.CO/2002.10771].

[70] Y. Ali-Haïmoud, E. D. Kovetz and M. Kamionkowski, Phys. Rev. D 96, no. 12, 123523 (2017) [astro-ph.C0/1709.06576].

[71] M. Raidal, C. Spethmann, V. Vaskonen and H. Veermäe, JCAP 02 (2019), 018 [astro-ph.C0/1812.01930].

[72] V. Vaskonen and H. Veermäe, Phys. Rev. D 101 (2020) no.4, 043015 [astro-ph.C0/1908.09752].

[73] V. De Luca, G. Franciolini, P. Pani and A. Riotto, Phys. Rev. D 102 (2020) no.4, 043505 [astro-ph.CO/2003.12589].

[74] V. De Luca, G. Franciolini, P. Pani and A. Riotto, JCAP 06 (2020), 044 [astro-ph.C0/2005.05641].

[75] A. Katz, J. Kopp, S. Sibiryakov and W. Xue, JCAP 12 (2018), 005 [astro-ph.Co/1807.11495].

[76] P. Montero-Camacho, X. Fang, G. Vasquez, M. Silva and C. M. Hirata, JCAP 08 (2019), 031 [astro-ph.C0/1906.05950].

[77] L. Lentati et al., Mon. Not. Roy. Astron. Soc. 453, no. 3, 2576 (2015) [astro-ph. CO/1504.03692].

[78] R. M. Shannon et al., Science 349, no. 6255, 1522 (2015) [astro-ph.C0/1509.07320].

[79] Z. Arzoumanian et al. [NANOGRAV], Astrophys. J. 859 (2018) no.1, 47 [astro-ph.HE/1801.02617].

[80] K. Aggarwal et al., Astrophys. J. 880 (2019), 2 [astro-ph.GA/1812.11585].

[81] W. Zhao, Y. Zhang, X. P. You and Z. H. Zhu, Phys. Rev. D 87, no. 12, 124012 (2013) [astro-ph.Co/1303.6718].

[82] C. Caprini et al., JCAP 1604, no. 04, 001 (2016) [astro-ph.C0/1512.06239].

[83] K. Yagi and N. Seto, Phys. Rev. D 83, 044011 (2011) Erratum: [Phys. Rev. D 95, no. 10, 109901 (2017)] [astro-ph.CO/1101.3940].

[84] B. P. Abbott et al. [LIGO Scientific Collaboration], Class. Quant. Grav. 34, no. 4, 044001 (2017) [astro-ph. IM/1607.08697].

[85] C. J. Moore, R. H. Cole and C. P. L. Berry, Class. Quant. Grav. 32 (2015) no.1, 015014 [gr-qc/1408.0740].

[86] B. S. Sathyaprakash and B. F. Schutz, Living Rev. Rel. 12 (2009) 2 [gr-qc/0903.0338]; Einstein Telescope, design at http://www.et-gw.eu/. 
[87] B. P. Abbott et al. [LIGO Scientific and Virgo Collaborations], Phys. Rev. Lett. 118 (2017) no.12, 121101 Erratum: [Phys. Rev. Lett. 119 (2017) no.2, 029901] [gr-qc/1612.02029].

[88] J. Coleman [MAGIS-100 Collaboration], [physics.ins-det/1812.00482].

[89] Y. A. El-Neaj et al. [AEDGE], EPJ Quant. Technol. 7 (2020), 6 [gr-qc/1908.00802].

[90] L. Badurina, E. Bentine, D. Blas, K. Bongs, D. Bortoletto, T. Bowcock, K. Bridges, W. Bowden, O. Buchmueller and C. Burrage, et al. JCAP 05 (2020), 011 [astro-ph.C0/1911.11755].
[91] A. Kusenko, M. Sasaki, S. Sugiyama, M. Takada, V. Takhistov and E. Vitagliano, Phys. Rev. Lett. 125 (2020) no.18, 18 [astro-ph.C0/2001.09160].

[92] A. Sesana, F. Haardt, P. Madau and M. Volonteri, Astrophys. J. 611 (2004), 623-632 [astro-ph/0401543].

[93] S. Blasi, V. Brdar and K. Schmitz, [astro-ph.CO/2009.06607].

[94] J. Ellis and M. Lewicki, [astro-ph.C0/2009.06555].

[95] Ville Vaskonen and Hardi Veermäe, [astro-ph.CO/2009.07832]

[96] S. Sugiyama, V. Takhistov, E. Vitagliano, A. Kusenko, M. Sasaki and M. Takada, [astro-ph.CO/2010.02189]. 